\title{
Hedging under Gamma constraints by optimal stopping and face-lifting
}

\author{
H. Mete Soner* \\ Koç University \\ Istanbul, Turkey \\ msoner@ku.edu.tr
}

\author{
Nizar Touzi \\ CREST and CEREMADE \\ Paris, France \\ touzi@ensae.fr
}

first version: March 2005, this version: September 2, 2005

\begin{abstract}
A super-replication problem with a gamma constraint, introduced in [12], is studied in the context of the one-dimensional Black and Scholes model. Several representations of the minimal super-hedging cost are obtained using the characterization derived in [3]. It is shown that the upper bound constraint on the gamma implies that the optimal strategy consists in hedging a conveniently face-lifted payoff function. Further an unusual connection between an optimal stopping problem and the lower bound is proved. A formal description of the optimal hedging strategy as a succession of periods of classical Black-Scholes hedging strategy and simple buy-and-hold strategy is also provided.
\end{abstract}

Key words : hedging under constraints, stochastic control, optimal stopping.

MSC 2000 subject classifications: Primary 93E20, 35C15; secondary 35D99.

JEL classification: G11, G13.

${ }^{*}$ Member of the Turkish Academy of Sciences and this work was partly supported by the Turkish Academy of Sciences. 


\section{Introduction}

There are many financial markets in which all contingent claims can not be perfectly hedged. During the past two decades several approaches for pricing and hedging in these models have been developed. One of these is the method of super-hedging and it requires the investor to be risk free at maturity by having a final wealth at least as large as the option liability. This fact makes it independent of the preferences of the investor and as such this approach provides prices and hedging strategies that are universal in nature. Although this is the main attractive feature of super-hedging, it is its chief weakness as well. It is believed that the super-hedging criterion is so stringent that the premium to be paid for being completely risk-free is too large. While this statement is shown to be correct for some models [11], there are several interesting classes of problems for which the super-hedging premium is small. Portfolio constraints with not so tight bounds is such a model with a simple explicit solution $[2,5]$. Clearly for these models, super-hedging provides a strong alternative. In addition to its practical use in some cases, the superreplication value is of theoretical importance as it represents the minimal investment in order to hedge without risk. In particular, the minimal super-hedging price is the upper value of the interval of all no-arbitrage prices. The lower value of this interval is the dual of the super-replication premium for the buyer viewpoint [10].

In this paper, we continue our study of the problem of super-replication under gamma constraints. This question was first raised in [2] and was later formulated precisely in [12]. A characterization of the minimal price as the unique solution of a non-standard dynamic programming equation was obtained in [3]. In this paper, we obtain a complete description for the one-dimensional Black-Scholes model. In particular, we prove several different characterizations through classical stopping time and control problems as described later in this Introduction. In the final section, we formally construct a hedge with bounded sensitivity parameters using these new equivalent formulations of the problem.

To explain the gamma constraint, we revisit the classical Black-Scholes framework with one riskless asset, normalized to unity, and one risky asset whose price process evolves according to the stochastic differential equation $d S(t)=\sigma S(t) d W(t)$, after a convenient change of measure. Then, the unconstrained super-replication cost of a European contingent claim of the type $g(S(T))$, is given by the Black-Scholes arbitrage price $v^{B S}(t, s)$ $=\mathbb{E}[g(S(T)) \mid S(t)=s]$. The optimal hedging strategy consists of holding $\Delta(t, S(t)):=$ $v_{s}^{B S}(t, S(t))$ units of the risky asset at each time $t \in[0, T]$. The variation of this optimal hedging strategy is an important problem that options traders face in practice and it is measured by the Gamma coefficient. It is defined by $\gamma(t, S(t)):=v_{s s}^{B S}(t, S(t))$. Since traders only act in discrete-time, a large $\gamma$ induces an important risk exposure between two transaction dates. Therefore, it is important to define hedging strategies with bounds on the Gamma. Also it is well known that the sensitivity parameter with respect to volatility, 
Vega, is an integral of Gamma. Hence, bounding Gamma also yields a bound for Vega as well.

This problem was raised by Broadie, Cvitanić and Soner [2] who provided an upper bound for its value. A precise description of the model under upper gamma constraint was then introduced by Soner and Touzi [12]. The analysis of the model was made possible by appealing to an original dynamic programming principle, which turned out to be a powerful tool for the treatment of general target problems. In the context of the one-dimensional Black-Scholes model, the solution of the super-replication problem under upper gamma constraint was described precisely in [12]. It consists in hedging a conveniently face-lifted payoff function according to the classical Black-Scholes hedging strategy.

The technical problems caused by the lower bound on the gamma were overcome later in [3] and [4] in a general multi-dimensional model. A brief description of these results in the one-dimensional framework is presented in Section 2. An interesting feature of this problem is that the associated Bellman equation is not the intuitive one. This is in contrast with usual stochastic control problems. The super-replication value was characterized in [3] as the unique viscosity solution of the associated Bellman equation with terminal condition given by the face lifted payoff. An important observation is that the face lifting is only driven by the upper bound on the Gamma.

In the one-dimensional framework a simpler characterization is available. The chief result of this paper is the following representation of the minimal super-replication price as the value of an optimal stopping problem. Let $V$ be this price under the constraint (2.5):

$$
-\underline{\Gamma} \leq S^{2}(t) \gamma(t, S(t)) \leq \bar{\Gamma}
$$

Precise definitions of $V$ and $\gamma$ are given in Section 2.3 and respectively, in Section 2.2 below. A discussion of different possible forms of the gamma constraint is also given in that section. In this paper, we do not provide any mechanism for choosing the upper and lower bounds that appear in the above constraint. However, in practice one can compute the premium attached to these bounds. That is the difference between the Black-Scholes price and the super-replication price. Indeed, in classical examples such as Call, Put and Digital options with only upper bound, this premium is explicitly available [10]. Then, an optimal choice of the bound can be made by balancing the premium due to the bound and the advantage obtained by having a bounded Gamma.

The main representation results of this paper are formulated in the following result. 
Theorem 1.1 Let $V$ be the minimal super-replicating price of a contingent claim with pay-off $g$. Assume $g$ satisfies (2.6). Then, for all $t<T$,

$$
\begin{aligned}
V(t, s) & =\sup _{\theta \in \mathcal{T}_{t}^{T}} \sup _{\tau \in \mathcal{T}_{\theta}^{\infty}} \mathbb{E}\left[g\left(S_{t, s}(\tau)\right)-\frac{1}{2} \sigma^{2}(\bar{\Gamma}(\tau-\theta)+\underline{\Gamma}(T-\theta))\right], \\
& =\sup _{\theta \in \mathcal{T}_{t}^{T}} \mathbb{E}\left[\hat{g}\left(S_{t, s}(\theta)\right)-\frac{1}{2} \sigma^{2} \underline{\Gamma}(T-\theta)\right], \\
& =\sup _{x \in \mathcal{X}} \mathbb{E}\left[-\frac{1}{2} \underline{\Gamma} \sigma^{2} \int_{t}^{T} x(r) d r+\hat{g}\left(S_{t, s}^{x}(T)\right)\right], \\
& \left.=\sup _{(x, y) \in \mathcal{X} \times \mathcal{Y}} \mathbb{E}\left[-\frac{1}{2} \sigma^{2} \int_{t}^{T}(\underline{\Gamma} x(r)+\bar{\Gamma} y(r)) d r\right]+\hat{g}\left(\hat{S}_{t, s}^{x, y}(T)\right)\right],
\end{aligned}
$$

where for any stopping times $0 \leq a \leq b \leq \infty, \mathcal{T}_{a}^{b}$ is the set of all $\mathbb{F}$-stopping times with values in the stochastic interval $[a, b], \hat{g}$ is the face-lifted terminal function given in (3.2) below. $\mathcal{X}$ and $\mathcal{Y}$ are the sets of all progressively measurable processes taking values in $[0,1]$, and, respectively, in $\mathbb{R}^{+}$. Finally, $S^{x}=\hat{S}^{x, 0}$, and the process $\hat{S}^{x, y}$ is defined by

$$
\hat{S}_{t, s}^{x, y}(t)=s, \quad d \hat{S}_{t, s}^{x, y}(r)=\hat{S}_{t, s}^{x, y}(r) \sigma \sqrt{1-x(r)+y(r)} d W(r) .
$$

This theorem is proved in Sections 5 and 6 below.

Our analysis of this problem in this and previous papers rely on the theory of viscosity solutions. A convex duality approach, which is generally available in most financial optimization problems, is not yet available for the gamma constraint. The above characterizations, especially the representation through the optimal stopping problem, may prove to be useful in this direction. Also these different characterizations enable us to provide a formal hedging strategy.

The starting point of our analysis is the dynamic programming equation of [3]. This equation is defined as the parabolic envelope of the first guess operator. Section 3 outlines the properties of this equation in the one-dimensional framework. This leads to a representation for the minimal super-replication value as the value function of a standard optimal control problem which is the final representation (1.4) in the above theorem. This characterization can be seen as the dual formulation of the problem along the lines of the literature on the super-replication problem under portfolio constraints (see [8] and the references therein). Indeed, the control problem (1.4) is the supremum of conveniently penalized Black-Scholes values of the contingent claim $g(S(T))$. However, in contrast with the problem of portfolio constraints, the penalty in (1.4) is in the volatility parameter. This indicates that the duality result can not simply be proved through classical change of measure techniques.

In Section 5, we show that the stochastic representation simplifies considerably, by a separating the treatment of both sides of the constraints in the context of the Black-Scholes 
model. More precisely, once the payoff is modified or face-lifted, one can forget about the upper bound on the gamma, and work only to satisfy the lower bound on the gamma. This is the representation (1.3) in the main theorem. Given this simplified stochastic representation, we then show in Section 6 that the value of super-replication coincides with the problem of optimal stopping the process $\hat{g}(S(t))-\frac{1}{2} \sigma^{2} \underline{\Gamma}(T-t)$, where $\hat{g}$ is the face-lifted payoff, and $\underline{\Gamma}$ is the lower bound on the gamma. This is the representation (1.2) in the main theorem. The first representation (1.1) follows from the observation that the face-lifted payoff is the value function of an infinite horizon optimal stopping problem.

Section 7 is devoted to the description of the optimal hedging strategy. We show that it is optimal to buy-and-hold the risky asset inside the domain where the lower bound on gamma is violated by the value function, and to apply the Black-Scholes strategy otherwise. In the final section, we list representation results under a similar gamma constraint.

\section{Hedging under Gamma constraints in the Black-Scholes model}

In this section, we formulate the problem of super-replication under upper and lower Gamma constraints, and recall the characterization of this problem in terms of the associated dynamic programming equation. This is essentially a reduction of the general framework of [3] to the one-dimensional Black-Scholes model.

\subsection{Model formulation}

The probabilistic structure of the model is the classical canonical filtered space of continuous functions equipped with the Wiener measure. The generic point $\omega=\omega(.) \in \Omega$ of this space is a continuous function on $\mathbb{R}_{+}$with $\omega(0)=0$. We denote by $\mathcal{F}(t)$ the $\sigma$-algebra generated by the family $\{\omega(s), s \leq t\}$ augmented by all $P$-null sets of $\Omega$. It is well-known that $\mathcal{F}(t)=\cap_{\varepsilon>0} \mathcal{F}(t+\varepsilon)$.

This defines a filtered probability space $(\Omega, \mathcal{F}, \mathbb{F}, P)$ carrying a one-dimensional standard Brownian motion $\{W(t), 0 \leq t \leq T\}$, with $\mathcal{F}(t)$ the smallest filtration that contains the filtration generated by $\{W(t), t \geq 0\}$ and satisfying the usual assumptions.

We consider a financial market which consists of a cash account and one risky asset. We take the cash account as numéraire and assume that the prices of the risky assets evolve according to the Black and Scholes model

$$
d S(r)=S(r) \sigma d W(r)
$$

where $\sigma$ is a positive constant. We shall denote by $S_{t, s}($.$) the solution of this equation$ 
with initial data $S(t)=s$, i.e.

$$
S_{t, s}(r):=s \exp \left[-\frac{\sigma^{2}}{2}(r-t)+\sigma(W(r)-W(t))\right] \quad \text { for } \quad(t, s) \in[0, T] \times \mathbb{R}_{+} .
$$

Observe that the restriction of the price process $S$ to a martingale does not induce any loss of generality. Indeed, we can reduce an initially drifted price process into a local martingale under an equivalent probability measure, by the usual Girsanov transformation. Since our problem of interest, which will be defined in (2.7) in the subsequent $\S 2.3$, involves the reference measure only through the associated family of zero measure sets, the problem is not changed by moving from $P$ to any equivalent measure.

\subsection{Trading strategies and gamma constraints}

Consider an economic agent that starts at time $t$ with initial capital $x$ and holds $Y(r)$ shares of the risky asset $S$ at time $r \in[t, T]$. Assume that the stochastic integral $\int_{t}^{r} Y(u) d S_{t, s}(u)$, $r \in[t, T]$, exists and trading is done in a self-financing way. Then, the evolution of the economic agent's wealth is given by

$$
X(r)=x+\int_{t}^{r} Y(u) d S_{t, s}(u) \text { for } \quad r \in[t, T] .
$$

To introduce constraints on the variations of investment strategies $Y$, we require them to be of the form

$$
Y(r)=\sum_{n=0}^{N-1} y_{n} \mathbf{1}_{\left\{\tau_{n} \leq r<\tau_{n+1}\right\}}+\int_{t}^{r} \alpha(u) d u+\int_{t}^{r} \gamma(u) d S_{t, s}(u),
$$

where $y_{0} \in \mathbb{R},\left\{\tau_{n}\right\}_{n \geq 0}$ is a non-decreasing sequence of $[t, T]$-valued stopping times such that $P$-almost surely, the random variable $N:=\inf \left\{n \in \mathbb{N}: \tau_{n}=T\right\}$ is almost surely bounded by a deterministic constant, and $t=\tau_{0}<\tau_{1}<\tau_{2}<\cdots<\tau_{N}=T$; for $n \geq 1$, $y_{n}$ is an real-valued, $\mathcal{F}_{\tau_{n}}$-measurable bounded random variable; $\alpha$ is a predictable process taking values in $\mathbb{R}$; and $\gamma$ is a real-valued stochastic process of the form

$$
\gamma(r)=\sum_{n=0}^{N-1} z_{n} \mathbf{1}_{\left\{\tau_{n} \leq r<\tau_{n+1}\right\}}+\int_{t}^{r} \gamma_{1}(u) d u+\int_{t}^{r} \gamma_{2}(u) d W(u),
$$

where $z_{0} \in \mathbb{R}$; for $n \geq 1, z_{n}$ is an $\mathcal{F}_{\tau_{n}}$-measurable random variable; and $\gamma_{1}$ and $\gamma_{2}$ are predictable processes taking values in $\mathbb{R}$. We assume that all above processes $Y, \alpha, \gamma, \gamma_{i}$ at any time to be bounded by a polynomial function of the stock price at that time, i.e, we assume that there exists an integer $k \geq 0$ and a constant $C \geq 0$ so that for all $r \in[t, T]$,

$$
|Y(r)|+|\alpha(r)|+|\gamma(r)|+\left|\gamma_{i}(r)\right| \leq C\left[1+|S(r)|^{k}\right] .
$$

It is also standard to impose a lower bound on the wealth process to exclude doubling strategies. However, we do not need this assumption in addition to previous technical conditions. 
Under these assumptions, a trading strategy is determined by the control

$$
\nu:=\left(\left(\tau_{n}, y_{n}\right)_{n \geq 0}, \alpha, \gamma\right)
$$

The set of admissible controls $\mathcal{A}_{t, s, x}$ is the collection of all such controls which obey in addition to the following gamma constraint:

$$
-\underline{\Gamma} \leq S_{t, s}(r)^{2} \gamma(r) \leq \bar{\Gamma} \text { for all } r \in[t, T],
$$

where $-\underline{\Gamma}<0<\bar{\Gamma}$ are two given constants. The particular choice of this constraint is discussed below. Other type of similar constraints can also be handled by our techniques, yielding essentially the same results, see Section 8. By

$$
\left\{\left(X_{t, s, x}^{\nu}, Y_{t, s}^{\nu}\right)(r), t \leq r \leq T\right\}
$$

we denote the process defined by the dynamics (2.2) and (2.3), the initial condition $X_{t, s, x}^{\nu}(t)=x$ and the control $\nu \in \mathcal{A}_{t, s, x}$.

\section{Discussion.}

1. By (2.3) the process $\gamma$ corresponds to the variation of the $Y$ process with respect to the stock price changes. Since $Y$ is the number of stock holdings at a given time, it is dimensionless and therefore, the quantity $S^{2} \gamma$ has the units of the stock price. Also, as we will see, in terms of the price $V, \gamma$ is formally equal to $V_{s s}$. This formal argument also leads to the same dimension analysis. Therefore, the constraint (2.5) is an attempt to control a quantity which has the units of money.

However, it may be desirable to bound dimensionless quantities. Indeed, in the portfolio constraint one controls the ratio, $s V_{s} / V$, a dimensionless quantity. In this context, it may be better to bound either $s V_{s s}$ or $s^{2} V_{s s} / V$. These two quantities are controlled, respectively, by $S_{t, s}(r) \gamma(r)$ and $S_{t, s}(r)^{2} \gamma(r) / X(r)$. The first constraint was studied in our first paper [12]. We shall comment on the latter constraint in Section 8. The choice of the constraint (2.5) is mainly motivated by our desire to simplify the exposition.

2. The problem of hedging under upper gamma constraints was first introduced in [12] under a slightly simpler formulation :

(i) first, instead of defining the process $\gamma$ by (2.4), it was taken directly as a control process,

(ii) secondly, there were no jumps allowed in the definition of the process $Y$.

These additional complications in the model have been introduced in [3] in order to formulate general constraints on the gamma, i.e. upper and lower bounds in our one-dimensional model. The formulation of the process $\gamma$ by (2.4) was needed in order to fit into the conditions of Theorem 3.5 in [4] which describes the small time path behavior of double stochastic integrals. As for the jumps introduced in the definition of the process $Y$, this relaxation was needed in order to state a dynamic programming principle. 


\subsection{Value function}

Throughout this paper, we shall consider a function $g: \mathbb{R}_{+} \longrightarrow \mathbb{R}$, and we define the European contingent claim $g\left(S_{t, s}(T)\right)$. We allow for a large class of such contingent claims by requiring the following mild condition on $g$ :

$$
g \text { is non-negative, lower semi-continuous, and } \sup _{s \geq 0} g(s) /[1+s]<\infty .
$$

The chief goal of this paper is to solve explicitly the super-replication problem of $g$ under the gamma constraint (2.5), i.e.

$$
V(t, s):=\inf \left\{x \in \mathbb{R}: X_{t, s, x}^{\nu}(T) \geq g\left(S_{t, s}(T)\right) \text { for some } \nu \in \mathcal{A}_{t, s, x}\right\},
$$

where we set $\inf \emptyset:=\infty$.

\section{Face lifting and HJB characterization}

The associated dynamic programming equation was derived in [3] and [4] in a more general multi-dimensional context. Here we report these results in one dimension (i.e., one underlying stock).

The minimal super-replication price $V$ is proved (c.f. [3]) to be the viscosity solution of the following equation,

$$
\hat{F}\left(V_{t}(t, s), s^{2} V_{s s}(t, s)\right)=0 \quad \text { on } \quad[0, T) \times \mathbb{R}_{+},
$$

where

$$
\hat{F}(p, A):=\sup _{\beta \geq 0} F(p, A+\beta) \text { and } F(p, A):=\min \left\{-p-\frac{1}{2} \sigma^{2} A, \bar{\Gamma}-A, \underline{\Gamma}+A\right\} .
$$

The operator $\hat{F}$ is called the parabolic envelope of $F$, as it is shown in [3] that $\hat{F}(p, A)$ is the smallest majorant of $F(p, A)$ which is non-increasing in the $A$ variable.

To formulate the final data in the gamma constrained problem, we define the face-lifted payoff function by

$$
\hat{g}(s):=h^{\mathrm{conc}}(s)-\bar{\Gamma} \ln s \quad \text { where } \quad h(s):=g(s)+\bar{\Gamma} \ln s,
$$

and $h^{\text {conc }}$ denotes the concave envelope of $h$. The function $\hat{g}$ appears naturally as the smallest majorant of the payoff function $g$ such that $s \longmapsto \hat{g}(s)+\bar{\Gamma} \ln s$ is concave, which is the weak formulation of the upper Gamma constraint $s^{2} \hat{g}_{s s} \leq \bar{\Gamma}$. Observe that $\hat{g}$ is finite under the affine growth condition assumed in (2.6).

Before stating the main HJB characterization of the super-replication problem $V$ from [3], we provide some examples which show that the face-lifted function $\hat{g}$ can be computed explicitly in all relevant examples. 
Example 3.1 (European call option) Consider the case $g(s):=(K-s)^{+}$for some $K>0$. Then, we directly compute that

$$
\hat{g}(s)= \begin{cases}0 ; & s \leq s_{0} \\ \bar{\Gamma} \ln \left(\frac{s_{0}}{s}\right)+\bar{\Gamma}\left(\frac{s_{0}}{s}-1\right) ; & s_{0} \leq s \leq s_{1} \\ s-K ; & s \geq s_{1},\end{cases}
$$

where

$$
s_{1}:=\left(\frac{1}{s_{0}}-\frac{1}{\bar{\Gamma}}\right)^{-1},
$$

and $s_{0}$ is defined by

$$
0<s_{0}<K \wedge \bar{\Gamma} \text { and } \ln \left(1-\frac{s_{0}}{\bar{\Gamma}}\right)+\frac{K}{\bar{\Gamma}}=0 .
$$

Example 3.2 (Digital option) Consider the case $g(s):=\mathbf{1}_{s \geq K}$ for some $K>0$. Then we directly compute that

$$
\hat{g}(s)= \begin{cases}0 ; & s \leq s_{0} \\ \bar{\Gamma} \ln \left(\frac{s_{0}}{s}\right)+\bar{\Gamma} \frac{s-s_{0}}{s_{0}} ; & s_{0} \leq s \leq 1 \\ 1 ; & s \geq 1\end{cases}
$$

where $s_{0}$ is defined by

$$
0<s_{0}<K \text { and } \ln \left(\frac{s_{0}}{K}\right)+\left(\frac{K}{s_{0}}-1\right)-\frac{1}{\bar{\Gamma}}=0
$$

By definition $V(T,)=$.$g . However, it is well-known that, in general, the presence of$ portfolio constraints implies that the value function is larger than the payoff function $g$ when the time to maturity approaches zero. This was first proved by Broadie, Cvitanić and Soner [2]. To make this statement precise, set

$$
\hat{V}(t, .):=V(t, .) \text { for } t<T \text { and } \hat{V}(T, .)=\hat{g} .
$$

The following theorem states, in particular, that the final data achieved by the value function is indeed equal to $\hat{g}$.

Theorem 3.1 ([3]) Assume that $g$ satisfies (2.6), and let $\bar{\Gamma}$ be a finite given upper bound on the gamma process. Assume further that

$$
\text { either } \underline{\Gamma}<\infty \text { or } h^{\text {conc }}(s)-\alpha s^{\gamma} \text { is convex for some } \alpha>0, \gamma>1 \text {. }
$$

Then, there exists a constant $C>0$ such that

$$
\sup _{s \geq 0}|\hat{V}(t, s)-\hat{g}(s)| \leq C(T-t) \quad \text { for all } t \in[0, T],
$$

and $\hat{V}$ is the unique viscosity solution to the equation (3.1) satisfying (3.4). In particular $\hat{V}$ is continuous on $[0, T] \times \mathbb{R}_{+}$. 
The uniqueness statement in Theorem 3.1 was obtained as a consequence of the following comparison result.

Proposition 3.1 ([3]) Assume that $g$ satisfies (2.6). Suppose that $\underline{w}$ is an upper semicontinuous viscosity sub-solution of (3.1), and $\bar{w}$ is a lower semi-continuous viscosity supersolution of (3.1). Further, assume that there exists a constant $C$ so that

$$
\underline{w}(t, s) \leq \hat{g}(s)+C(T-t) \quad \text { and } \quad \bar{w}(t, s) \geq \hat{g}(s)-C(T-t)
$$

for all $(t, s) \in[0, T) \times \mathbb{R}_{+}$. Then,

$$
\underline{w} \leq \bar{w} \quad \text { on } \quad[0, T] \times \mathbb{R}_{+} .
$$

Observe that the statement of Proposition 3.1 holds for every $-\infty \leq-\underline{\Gamma}<0<\bar{\Gamma} \leq \infty$.

Example 3.3 (The case $\underline{\Gamma}=\infty$ ) This case was considered in [12] under a slightly different formulation of the upper bound constraint on the gamma process. When the control process $\gamma$ is only subject to an upper bound constraint, the operator $F$ is parabolic, and

$$
\hat{F}(p, A)=F(p, A)=\min \left\{-\hat{V}_{t}-\frac{1}{2} \sigma^{2} s^{2} \hat{V}_{s s}, \bar{\Gamma}-s^{2} \hat{V}_{s s}\right\}=0 .
$$

Under the condition

$$
s \longmapsto h^{\text {conc }}(s)-\alpha s^{\gamma} \text { is convex for some } \alpha>0, \gamma>1,
$$

it follows that

$$
\hat{V}(t, s)=v(t, s):=\mathbb{E}\left[\hat{g}\left(S_{t, s}(T)\right)\right], \text { i.e. }
$$

the value function of the super-replication problem under upper gamma constraint is given by the Black-Scholes price of the face-lifted payoff $\hat{g}$. To see this, observe that

$$
\begin{aligned}
v(t, s)+\bar{\Gamma} \ln s & =\mathbb{E}\left[h^{\text {conc }}\left(S_{t, s}(T)\right)-\bar{\Gamma} \ln S_{t, s}(T)\right]+\bar{\Gamma} \ln s \\
& =\mathbb{E}\left[h^{\text {conc }}\left(S_{t, s}(T)\right)\right]-\frac{1}{2} \sigma^{2}(T-t) .
\end{aligned}
$$

By the concavity of $h^{\text {conc }}$ and the smoothness of $v$, this implies that $\bar{\Gamma}-s^{2} v_{s s} \geq 0$. Since $-v_{t}-\frac{1}{2} \sigma^{2} s^{2} v_{s s}=0$, we deduce that $v$ is a classical solution of the PDE (3.1). Under the condition (3.5), it is easily checked that $v(t, s) \geq \hat{g}(s)-C(T-t)$ for some constant $C$ independent of $(t, s)$. We then conclude that (3.6) holds by the uniqueness result of Proposition (3.1).

In the rest of this paper, we focus on the case where the lower bound $-\underline{\Gamma}$ is finite. The first step is the following explicit characterization of the operator $\hat{F}(p, A)=0$ which holds in the multidimensional models as well. Let

$$
G(p, A):=\min \left\{-p-\frac{1}{2} \sigma^{2}\left[(A+\underline{\Gamma})^{+}-\underline{\Gamma}\right], \bar{\Gamma}-A\right\} .
$$


Lemma 3.1 For all $(p, A) \in \mathbb{R}^{2}$, we have $\hat{F}(p, A) \geq 0 \quad$ (respectively, $\leq 0$ ) if and only if $G(p, A) \geq 0(\leq 0)$.

Proof. It is sufficient to prove that $\hat{F}(p, A) \geq 0$ iff $G(p, A) \geq 0$.

1. Suppose that $\hat{F}(p, A) \geq 0$. Since $\lim _{\beta \rightarrow \infty} \mathbb{F}(p, A+\beta)=-\infty$, there exists $\hat{\beta} \geq 0$ such that $0 \leq \hat{F}(p, A)=F(p, A+\hat{\beta})$. Then, we directly verify that $G(p, A+\hat{\beta}) \geq 0$. Since $G$ is non-increasing in the $A$ variable, this implies that $G(p, A) \geq 0$.

2. Conversely, assume that $G(p, A) \geq 0$, i.e.

$$
\bar{\Gamma}-A \geq 0 \text { and }-p-\frac{1}{2} \sigma^{2}\left[(A+\underline{\Gamma})^{+}-\underline{\Gamma}\right] .
$$

Set $\beta:=(A+\underline{\Gamma})^{+}-(A+\underline{\Gamma}) \geq 0$ so that

$$
\begin{gathered}
\underline{\Gamma}+A+\beta=(A+\underline{\Gamma})^{+} \geq 0, \\
\underline{\Gamma}-(A+\beta)=\underline{\Gamma}+\bar{\Gamma}-(A+\underline{\Gamma})^{+}=\min \{\bar{\Gamma}+\underline{\Gamma}, \bar{\Gamma}-A\} \geq 0,
\end{gathered}
$$

and

$$
-p-\frac{1}{2} \sigma^{2}(A+\beta)=-p-\frac{1}{2} \sigma^{2}\left[(A+\underline{\Gamma})^{+}-\underline{\Gamma}\right] \geq 0 .
$$

Hence, $F(p, A+\beta) \geq 0$, and therefore $\hat{F}(p, A) \geq 0$.

\section{Formal PDE derivation of (1.3)}

In this section, we give a short intuitive PDE derivation of (1.3). This discussion provides a concise foundation for the underlying connections and may prove to be useful in other contexts as well. However, although this discussion can be turned into a rigorous proof with little extra effort, we choose to give a rigorous proof based on a probabilistic stochastic control argument. This proof will be given in the next section and it may apply to a larger class of models.

By Theorem 3.1 and Lemma 3.1, it follows that the value function $\hat{V}$ is the unique viscosity solution of the equation $G\left(\hat{V}_{t}, s^{2} \hat{V}_{s s}\right)=0$ on $[0, T) \times \mathbb{R}_{+}$satisfying (3.4). In order to derive a stochastic representation for $\hat{V}$, we observe that

$$
G(p, A)=\min \left\{\inf _{0 \leq x \leq 1}-p-\frac{1}{2} \sigma^{2} A(1-x)+\frac{1}{2} \underline{\Gamma} \sigma^{2} x, \bar{\Gamma}-A\right\}
$$

and

$$
G(p, A) \geq 0 \quad \Leftrightarrow \quad \inf _{0 \leq x \leq 1, y \geq 0}\left\{-p-\frac{1}{2} \sigma^{2}[1-x+y] A+\frac{1}{2} \sigma^{2}[\underline{\Gamma} x+\bar{\Gamma} y]\right\} \geq 0
$$


Let $U$ be the value function defined on the right hand side of (1.3) and

$$
\hat{U}(t, s):=\sup _{(x, y) \in \mathcal{X} \times \mathcal{Y}} \mathbb{E}\left[\hat{g}\left(\hat{S}_{t, s}^{x, y}(T)\right)-\frac{1}{2} \sigma^{2} \int_{t}^{T}(\underline{\Gamma} x(r)+\bar{\Gamma} y(r)) d r\right],
$$

where $\mathcal{Y}$ is the collection of all progressively adapted processes with values in $\mathbb{R}_{+}$, and the controlled process $\hat{S}_{t, s}^{x, y}$ is as in (1.5). Observe that $U$ corresponds to the control problem $\hat{U}$ with control process $y$ identically equal to zero.

One can show by classical techniques that the function $\hat{U}$ is a viscosity solution of the equation $G\left(\hat{U}_{t}, s^{2} \hat{U}_{s s}\right)=0$. Hence, by the uniqueness result Proposition 3.1 we deduce that $\hat{V}=\hat{U}$. We will not provide a proof of this claim as it will not be used in the subsequent analysis. Instead we assume it is formally proved and proceed to show that $\hat{U}=U$.

Since $h^{\text {conc }}(s):=\hat{g}(s)+\bar{\Gamma} \ln s$ is concave by the construction of $\hat{g}$, we claim that the optimal choice for the control process $y$ in the above optimization problem is $y \equiv 0$. Indeed, for all fixed controls $(x, y) \in \mathcal{X} \times \mathcal{Y}$, we compute that

$$
\begin{aligned}
& \mathbb{E}\left[\hat{g}\left(\hat{S}_{t, s}^{x, y}(T)\right)-\frac{1}{2} \sigma^{2} \int_{t}^{T}(\underline{\Gamma} x(r)+\bar{\Gamma} y(r)) d r\right] \\
= & \mathbb{E}\left[h^{\operatorname{conc}}\left(\hat{S}_{t, s}^{x, y}(T)\right)-\bar{\Gamma} \ln \hat{S}_{t, s}^{x, y}(T)-\frac{1}{2} \sigma^{2} \int_{t}^{T}(\underline{\Gamma} x(r)+\bar{\Gamma} y(r)) d r\right] \\
= & \mathbb{E}\left[h^{\operatorname{conc}}\left(\hat{S}_{t, s}^{x, y}(T)\right)-\frac{1}{2} \sigma^{2}(\bar{\Gamma}+\underline{\Gamma}) \int_{t}^{T} x(r) d r\right]-\bar{\Gamma}\left[\ln s+\frac{1}{2} \sigma^{2}(T-t)\right],
\end{aligned}
$$

so that for all control $x \in \mathcal{X}$, we are reduced to the problem of partial maximization with respect to $y$ :

$$
\sup _{y \in \mathcal{Y}} \mathbb{E}\left[h^{\operatorname{conc}}\left(\hat{S}_{t, s}^{x, y}(T)\right)\right] .
$$

By the scaling property of the Brownian motion, observe that

$$
\hat{S}_{t, s}^{x, y}(T)=_{\mathrm{d}} S_{t, s}\left(\theta^{x}+\theta^{y}\right) \quad \text { where } \theta^{x}:=t+\int_{t}^{T}(1-x(r)) d r \text { and } \theta^{y}:=\int_{t}^{T} y(r) d r,
$$

$S=\hat{S}^{0,0}$ is the original Black-Scholes process, and $=_{\mathrm{d}}$ denotes equality in distribution. Now, of course, $\theta^{x}$ and $\theta^{x}+\theta^{y}$ are not stopping times, but if they were and if the Doob optional sampling theorem applies, then it follows from the concavity of $h^{\text {conc }}$ that :

$$
\begin{aligned}
\mathbb{E}\left[h^{\text {conc }}\left(\hat{S}_{t, s}^{x, y}(T)\right)\right] & =\mathbb{E}\left[\mathbb{E}\left\{h^{\text {conc }}\left(S_{t, s}\left(\theta^{x}+\theta^{y}\right)\right) \mid \mathcal{F}_{\theta^{x}}\right\}\right] \\
& \leq \mathbb{E}\left[h^{\text {conc }}\left(S_{t, s}\left(\theta^{x}\right)\right)\right] \\
& =\mathbb{E}\left[h^{\text {conc }}\left(\hat{S}_{t, s}^{x, 0}(T)\right)\right]
\end{aligned}
$$

so that $y=0$ is the optimal process for the above partial maximization problem and $U=\hat{U}$.

The main ingredients in the above formal argument are the comparison result, which is standard in the PDE literature. The final step can be proved rigorously with extra care. 


\section{Stochastic representation in the standard form}

The chief result of this section is

Theorem 5.1 Assume that $g$ satisfies (2.6). Then the function $\hat{V}$ defined in (3.3) has the stochastic representation in standard form

$$
\hat{V}(t, s)=U(t, s):=\sup _{x \in \mathcal{X}} \mathbb{E}\left[\hat{g}\left(S_{t, s}^{x}(T)\right)-\frac{1}{2} \underline{\Gamma} \sigma^{2} \int_{t}^{T} x(r) d r\right],
$$

where the controlled process $S_{t, s}^{x}$ is defined by

$$
S_{t, s}^{x}(t)=s \quad \text { and } \quad d S_{t, s}^{x}(r)=S_{t, s}^{x}(r) \sigma \sqrt{1-x(r)} d W(r)
$$

and $\mathcal{X}$ is the collection of all progressively measurable processes with values in $[0,1]$.

We start the proof by obtaining the PDE characterization of $U$.

Lemma 5.1 The function $U$ is continuous and is the unique viscosity solution of the equation

$$
-U_{t}-\frac{1}{2} \sigma^{2}\left[\left(s^{2} U_{s s}+\underline{\Gamma}\right)^{+}-\underline{\Gamma}\right]=0 \quad \text { on } \quad[0, T) \times \mathbb{R}_{+},
$$

satisfying (3.4).

Proof. 1. Let $(t, s) \in[0, T) \times \mathbb{R}_{+}$, and $\theta$ be a stopping time with values in $[t, s]$. Then, the classical dynamic programming principle states that

$$
U(t, s)=\sup _{x \in \mathcal{X}} \mathbb{E}\left[U\left(\theta, S_{t, s}^{x}(\theta)\right)-\frac{1}{2} \underline{\Gamma} \sigma^{2} \int_{t}^{\theta} x(r) d r\right]
$$

see e.g. [6].

2. Observe that

$$
\inf _{0 \leq x \leq 1}\left\{-p-\frac{1}{2} \sigma^{2} A(1-x)+\frac{1}{2} \underline{\Gamma} \sigma^{2} x\right\}=-p-\frac{1}{2} \sigma^{2}\left[(A+\underline{\Gamma})^{+}-\underline{\Gamma}\right] .
$$

Observe that $U$ is locally bounded by Condition (2.6), and let $U_{*}$ and $U^{*}$ be, respectively, the lower and the upper semi-continuous envelopes of $U$, i.e.,

$$
U_{*}(t, s):=\liminf _{\left(t^{\prime}, s^{\prime}\right) \rightarrow(t, s)} U\left(t^{\prime}, s^{\prime}\right) \quad \text { and } \quad U^{*}(t, s):=\limsup _{\left(t^{\prime}, s^{\prime}\right) \rightarrow(t, s)} U\left(t^{\prime}, s^{\prime}\right) .
$$

Then, they are, respectively, viscosity super-solution and sub-solution of (5.1). This is proved by classical techniques as a consequence of the dynamic programming principle 
of Step 1, see e.g. [6]. By Lemma 3.1, it follows that $U_{*}$ and $U^{*}$ are also, respectively, super-solution and sub-solution of the equation

$$
\sup _{\beta \geq 0} \min \left\{-U_{t}-\frac{1}{2} \sigma^{2}\left[s^{2} U_{s s}+\beta\right], \underline{\Gamma}+s^{2} U_{s s}+\beta\right\}=0 \quad \text { on } \quad[0, T) \times \mathbb{R}_{+} .
$$

3. In this step, we prove that

$$
U_{*}(t, s) \geq \hat{g}(s)-\frac{1}{2} \sigma^{2} \underline{\Gamma}(T-t) \text { for all }(t, s) \in[0, T) \times \mathbb{R}_{+} .
$$

Indeed, since $U_{*}$ is a viscosity super-solution of the equation (5.1), by Lemma 3.1,

$$
\sup _{\beta \geq 0} \min \left\{-\left(U_{*}\right)_{t}-\frac{1}{2} \sigma^{2}\left[s^{2}\left(U_{*}\right)_{s s}+\beta\right], \underline{\Gamma}+s^{2}\left(U_{*}\right)_{s s}+\beta\right\} \geq 0
$$

in the viscosity sense. This implies that

$$
-\left(U_{*}\right)_{t}+\frac{1}{2} \sigma^{2} \underline{\Gamma} \geq 0 \quad \text { on } \quad[0, T) \times \mathbb{R}_{+},
$$

in the viscosity sense. Therefore,

$$
U_{*}(t, s)-U_{*}(T, s)+\frac{1}{2} \sigma^{2} \underline{\Gamma}(T-t) \geq 0 \text { for all }(t, s) \in[0, T] \times \mathbb{R}_{+} .
$$

By a direct passage to the limit in the definition of $U$ in the statement of the theorem, it follows from Fatou's lemma that

$$
U_{*}(T, s) \geq \hat{g}(s) \text { for all } s \in \mathbb{R}_{+} .
$$

We obtain (5.3) by combining the last two inequalities.

4. In this step, we prove that

$$
U(t, s) \leq \hat{g}(s)+\frac{1}{2} \bar{\Gamma} \sigma^{2}(T-t) \quad \text { for all } \quad(t, s) \in[0, T) \times \mathbb{R}_{+} .
$$

Recall that $\hat{g}(s)=h^{\text {conc }}(s)-\bar{\Gamma} \ln s$, where $h$ is given by (3.2), and $h^{\text {conc }}$ is the concave envelope of $h$. Since the control processes in $\mathcal{X}$ are bounded, Jensen's inequality yield

$$
\begin{aligned}
U(t, s) & =\sup _{x \in \mathcal{X}} \mathbb{E}\left[h^{\text {conc }}\left(S_{t, s}^{x}(T)\right)-\bar{\Gamma} \ln S_{t, s}^{x}(T)-\frac{1}{2} \sigma^{2} \underline{\Gamma} \int_{t}^{T} x(r) d r\right] \\
& =\frac{1}{2} \bar{\Gamma} \sigma^{2}(T-t)-\bar{\Gamma} \ln s+\sup _{x \in \mathcal{X}} \mathbb{E}\left[h^{\text {conc }}\left(S_{t, s}^{x}(T)\right)-\frac{1}{2} \sigma^{2}(\bar{\Gamma}+\underline{\Gamma}) \int_{t}^{T} x(r) d r\right] \\
& \leq \frac{1}{2} \bar{\Gamma} \sigma^{2}(T-t)+h^{\text {conc }}(s)-\bar{\Gamma} \ln s+\sup _{x \in \mathcal{X}} \mathbb{E}\left[-\frac{1}{2} \sigma^{2}(\bar{\Gamma}+\underline{\Gamma}) \int_{t}^{T} x(r) d r\right] \\
& =\frac{1}{2} \bar{\Gamma} \sigma^{2}(T-t)+\hat{g}(s)+\sup _{x \in \mathcal{X}} \mathbb{E}\left[-\frac{1}{2} \sigma^{2}(\bar{\Gamma}+\underline{\Gamma}) \int_{t}^{T} x(r) d r\right] \\
& =\frac{1}{2} \bar{\Gamma} \sigma^{2}(T-t)+\hat{g}(s),
\end{aligned}
$$


where the last equality follows from the fact that $\underline{\Gamma}+\bar{\Gamma} \geq 0$, together with the definition of the set of control processes $\mathcal{X}$.

5. Clearly $U^{*}$ inherits the bound (5.4) from $U$. Hence $U_{*}$ is a viscosity super-solution of the equation (5.2) satisfying (5.3), and $U^{*}$ is a viscosity sub-solution of the equation (5.2) satisfying (5.4), it follows from the comparison result of Theorem 3.1 that $U_{*} \geq U^{*}$. Since the reverse inequality holds true by the definition of these functions, we conclude that $U_{*}=U^{*}=U$. In particular, the function $U$ is continuous and satisfies

$$
|U(t, s)-\hat{g}(s)| \leq \frac{1}{2} \sigma^{2}(\underline{\Gamma} \vee \bar{\Gamma})(T-t) \quad \text { for all } \quad(t, s) \in[, T) \times \mathbb{R}_{+} .
$$

We are now in a position to prove the main result of this section which states that $\hat{V}=U$.

Proof of Theorem 5.1. We first observe that $\hat{V}$ is a viscosity super-solution of the equation (5.1). This follows from Theorem 3.1 together with Lemma 3.1. Since $U$ is a viscosity solution of this equation and satisfies the growth condition (3.4), we deduce from the comparison result of Proposition 3.1 that $U \leq \hat{V}$. In the remaining part of this proof, we show that the converse inequality holds.

1. We first prove that the function

$$
t \longmapsto U(t, s)+\frac{1}{2} \bar{\Gamma} \sigma^{2} t \text { is non-decreasing for all } s \in \mathbb{R}_{+} .
$$

By the dynamic programming principle stated in Step 1 of the proof of Lemma 5.1,

$$
U(t, s)=\sup _{x \in \mathcal{X}} \mathbb{E}\left[U\left(T-\eta, S_{t, s}^{x}(T-\eta)\right)-\frac{1}{2} \underline{\Gamma} \sigma^{2} \int_{t}^{T-\eta} x(r) d r\right]
$$

for all $0<\eta<T-t$. For an arbitrary parameter $\varepsilon>0$, let $\hat{x} \in \mathcal{X}$ be such that

$$
U(t, s) \leq \varepsilon+\mathbb{E}\left[U\left(T-\eta, S_{t, s}^{\hat{x}}(T-\eta)\right)-\frac{1}{2} \underline{\Gamma} \sigma^{2} \int_{t}^{T-\eta} \hat{x}(r) d r\right] .
$$

By the inequality (5.4) which was derived in Step 4 of the proof of Lemma 5.1,

$$
U(t, s) \leq \varepsilon+\frac{1}{2} \bar{\Gamma} \sigma^{2} \eta+\mathbb{E}\left[\hat{g}\left(S_{t, s}^{\hat{x}}(T-\eta)\right)-\frac{1}{2} \underline{\Gamma} \sigma^{2} \int_{t}^{T-\eta} \hat{x}(r) d r\right] .
$$

Since $\hat{x}$ is $\mathbb{F}$-progressively measurable, there is a measurable function $\phi$ such that

$$
\hat{x}(r):=\phi\left(r-t ; W_{u}, t \leq u \leq r\right) \text { for } t \leq r \leq T-\eta .
$$

Set

$$
\tilde{x}(r):=\phi\left(r-t-\eta ; W_{u}, t+\eta \leq u \leq r\right) \text { for } t+\eta \leq r \leq T,
$$


and observe that

$$
\int_{t}^{T-\eta} \hat{x}(r) d r=_{\mathrm{d}} \int_{t+\eta}^{T} \tilde{x}(r) d r \quad \text { and } \quad S_{t, s}^{\hat{x}}(T-\eta)=_{\mathrm{d}} S_{t+h, s}^{\tilde{x}}(T),
$$

where $=_{\mathrm{d}}$ denotes equality in distribution. We now rewrite the last inequality as

$$
\begin{aligned}
U(t, s) & \leq \varepsilon+\frac{1}{2} \bar{\Gamma} \sigma^{2} \eta+\mathbb{E}\left[\hat{g}\left(S_{t+\eta, s}^{\tilde{x}}(T)\right)-\frac{1}{2} \underline{\Gamma} \sigma^{2} \int_{t+\eta}^{T} \tilde{x}(r) d r\right] \\
& \leq \varepsilon+\frac{1}{2} \bar{\Gamma} \sigma^{2} \eta+U(t+\eta, s) .
\end{aligned}
$$

2. By Lemma 5.1,

$$
-U_{t}-\frac{1}{2} \sigma^{2} s^{2} U_{s s} \geq-U_{t}-\frac{1}{2} \sigma^{2}\left[\left(s^{2} U_{s s}+\underline{\Gamma}\right)^{+}-\underline{\Gamma}\right]=0
$$

in the viscosity sense. From the first step of this proof, it follows that $U_{t}+\frac{1}{2} \bar{\Gamma} \sigma^{2} \geq 0$ in the viscosity sense. Therefore,

$$
\bar{\Gamma}-s^{2} U_{s s} \geq 0 \text { on }[0, T) \times \mathbb{R}_{+} \text {in the viscosity sense. }
$$

This in turns implies that the function $U$ is a super-solution of the equation $G\left(U_{t}, s^{2} U_{s s}\right) \geq$ 0 , or equivalently by Lemma 3.1 ,

$$
\hat{F}\left(U_{t}, s^{2} U_{s s}\right) \geq 0 \quad \text { on }[0, T) \times \mathbb{R}_{+} \text {in the viscosity sense. }
$$

Since $U$ satisfies the growth condition (3.4), we deduce from the comparison result of Proposition 3.1 that $U \geq \hat{V}$. This completes the proof of the theorem.

\section{Optimal stopping the face-lifted payoff}

In this section, we provide an alternative representation of the super-replication value $\hat{V}$ in terms of an optimal stopping problem. The intuition behind this connection is that, for every $x \in \mathcal{X}$,

$$
\mathbb{E}\left[\hat{g}\left(S_{t, s}^{x}(T)\right)-\frac{1}{2} \underline{\Gamma} \sigma^{2} \int_{t}^{T} x(r) d r\right]=\mathbb{E}\left[\hat{g}\left(S_{t, s}\left(\theta^{x}\right)\right)-\frac{1}{2} \underline{\underline{\Gamma}} \sigma^{2}\left[(T-t)-\left(\theta^{x}-t\right)\right]\right],
$$

where $S_{t, s}=S_{t, s}^{0}$ is the underlying asset price process, and $\theta^{x}$ is the $[t, T]$-valued random variable defined by

$$
\theta^{x}:=t+\int_{t}^{T} x(r) d r
$$

The following result completes the proof of Theorem 1.1. 
Theorem 6.1 Assume that $g$ satisfies (2.6). Then the function $\hat{V}$ defined in (3.3) has the stochastic representation in standard form

$$
\begin{aligned}
\hat{V}(t, s) & =\sup _{\theta \in \mathcal{T}_{t}^{T}} \mathbb{E}\left[\hat{g}\left(S_{t, s}(\theta)\right)-\frac{1}{2} \sigma^{2} \underline{\Gamma}(T-\theta)\right] \\
& =\sup _{\theta \in \mathcal{T}_{t}^{T}} \sup _{\tau \in \mathcal{T}_{\theta}^{\infty}} \mathbb{E}\left[g\left(S_{t, s}(\tau)\right)-\frac{1}{2} \sigma^{2}(\bar{\Gamma}(\tau-\theta)+\underline{\Gamma}(T-\theta))\right],
\end{aligned}
$$

where $\mathcal{T}_{t}^{T}$ is the collection of all $\mathbb{F}$-stopping times with values in $[t, T]$.

Proof. Let

$w(t, s):=\sup _{\theta \in \mathcal{T}_{t}^{T}} \mathbb{E}\left[\hat{g}\left(S_{t, s}(\theta)\right)+\frac{1}{2} \underline{\Gamma} \sigma^{2}(\theta-t)\right]$ and $\hat{w}(t, s):=w(t, s)-\frac{1}{2} \underline{\Gamma} \sigma^{2}(T-t)$.

1. For every stopping time $\theta$ in $\mathcal{T}_{t}^{T}$, the process $\left\{x(r):=\mathbf{1}_{r \leq \theta}, t \leq r \leq T\right\}$ is in the family of controls $\mathcal{X}$. It then follows from the time change formula that

$$
-\underline{\Gamma} \frac{\sigma^{2}}{2}(T-t)+\mathbb{E}\left[\hat{g}\left(S_{t, s}(\theta)\right)-\frac{1}{2} \underline{\Gamma} \sigma^{2}(\theta-t)\right]=\mathbb{E}\left[\hat{g}\left(S_{t, s}^{x}(T)\right)-\frac{1}{2} \underline{\Gamma} \sigma^{2} \int_{t}^{T} x(r) d r\right],
$$

see e.g. [7]. Hence, $\hat{w} \geq \hat{V}$.

2. We now prove the converse inequality

$$
\hat{V} \geq \hat{w} .
$$

By classical techniques, see e.g. Lions [9], it follows that $w$ is a viscosity solution of the free boundary problem

$$
\min \left\{-w_{t}-\frac{1}{2} \sigma^{2} s^{2} w_{s s}+\frac{1}{2} \sigma^{2} \underline{\Gamma}, w-\hat{g}\right\}=0, \quad \text { on } \quad[0, T) \times \mathbb{R}_{+} .
$$

Then, the function $\hat{w}$ is a viscosity solution of the free boundary problem

$$
\min \left\{-\hat{w}_{t}-\frac{1}{2} s^{2} \sigma^{2} \hat{w}_{s s}, \hat{w}-\hat{g}+\frac{1}{2} \sigma^{2} \underline{\Gamma}(T-t)\right\}=0, \quad \text { on } \quad[0, T) \times \mathbb{R}_{+} .
$$

This equation satisfies a comparison result for semi-continuous functions with linear growth in $s$. We next observe that

$$
-\hat{V}_{t}-\frac{1}{2} \sigma^{2} s^{2} \hat{V}_{s s} \geq-\hat{V}_{t}-\frac{1}{2} \sigma^{2}\left[\left(s^{2} \hat{V}_{s s}+\underline{\Gamma}\right)^{+}-\underline{\Gamma}\right]=0
$$

in the viscosity sense. In view of (5.3), this shows that $\hat{V}$ is a viscosity super-solution of (6.2). Then, since $\hat{V}(T,)=.\hat{w}(T,),. \hat{V}$ satisfies (3.4) and $\hat{g}$ satisfies (2.6), the required inequality (6.1) follows from the comparison result.

3. It is well-known that the concave envelope of a function can be represented as the value function of an infinite horizon optimal stopping problem :

$$
h^{\text {conc }}(s)=\sup _{\tau \in \mathcal{T}_{0}^{\infty}} \mathbb{E}\left[h\left(S_{0, s}(\tau)\right)\right]
$$


We combine this with the already proved result that $\hat{V}=\hat{w}$. The result is

$$
\hat{V}(t, s)=\sup _{\theta \in \mathcal{T}_{t}^{T}, \tau \in \mathcal{T}_{\theta}^{\infty}} \mathbb{E}\left[g\left(S_{t, s}(\tau)\right)-\frac{1}{2} \sigma^{2}(\bar{\Gamma}(\tau-\theta)+\underline{\Gamma}(T-\theta))\right] .
$$

Remark 6.1 For future reference, we claim the following implication from Theorem 6.1 :

$$
\hat{V}(t, s)=\hat{g}(s)-\frac{1}{2} \sigma^{2} \underline{\Gamma}(T-t) \quad \text { whenever } s^{2} \hat{V}_{s s}(t, s)<-\underline{\Gamma} .
$$

This property is crucially important for the description of the hedging strategy. To prove (6.3), we recall that, by Lemma $3.1,-\hat{V}_{t}-\frac{1}{2} \sigma^{2}\left[\left(s^{2} \hat{V}_{s s}+\underline{\Gamma}\right)^{+}-\underline{\Gamma}\right]=0$. Now assume that $s^{2} \hat{V}_{s s}(t, s)<-\underline{\Gamma}$. Then $-\hat{V}_{t}-\frac{1}{2} \sigma^{2} s^{2} \hat{V}_{s s}>0$. This implies (6.3), since $\hat{V}$ satisfies the free boundary problem (6.2) by Theorem 6.1 .

\section{Formal hedging strategy under Gamma constraints}

In this section, we describe a formal optimal hedging strategy which satisfies the gamma constraint.

The subsequent analysis will be restricted to a formal discussion because in our discussion we will ignore some of the admissibility restrictions and assume some technical assumptions concerning the inflexion points of the value function.

\subsection{Reduction to a single non-negativity constraint on gamma}

Our previous results show that the upper bound $\bar{\Gamma}$ on the gamma process is easily treated by face-lifting the payoff, i.e. passing from $g$ to $\hat{g}$. Indeed, it follows from Theorem 5.1 that our super-replication problem reduces to the problem of hedging the face-lifted contingent claim with no upper bound on the gamma process. Then, the only non-trivial hedging problem is related to the presence of the lower bound $\underline{\Gamma}$ on the gamma process. In the rest of this section, we shall assume without loss of generality that $\bar{\Gamma}=\infty$.

We next show that, without loss of generality, we can reduce the problem to the case of a zero lower bound on the gamma process. To see this, let us decompose the payoff function $g$ into

$$
g(s)=\tilde{g}(s)+\ell(s) \text { where } \ell(s):=\underline{\Gamma} \ln s .
$$

Observe that the super-replication cost of the contingent claim $\ell\left(S_{T}\right)$ under the lower gamma constraint $\underline{\Gamma}$ is simply given by the Black-Scholes value

$$
\bar{V}(t, s):=\mathbb{E}\left[\underline{\Gamma} \ln S_{t, s}(T)\right]=\underline{\Gamma}\left(\ln s-\frac{1}{2} \sigma^{2}(T-t)\right) .
$$


This follows from the fact that the associated Black-Scholes strategy satisfies the lower bound on the gamma, as $s^{2} v_{s s}(t, s)=-\underline{\Gamma}$. Notice however that the BV process appearing in the dynamics of the portfolio process is given by $A(t)=\int_{0}^{t} \bar{\alpha}(r) d r$ with

$$
\bar{\alpha}(r)=\frac{1}{2} \sigma^{2} \frac{\underline{\Gamma}}{S(r)} .
$$

Although this process does not satisfy the admissibility conditions of Section 2, this technical difficulty can easily be circumvented by a convenient approximation.

Next, suppose that $(\tilde{y}, \tilde{A}, \tilde{\gamma})$ is a hedging strategy of the contingent claim $\tilde{g}\left(S_{T}\right)$ with zero lower bound on the gamma process. Then clearly, the aggregate strategy $(\bar{y}+\tilde{y}, \bar{A}+\tilde{A}, \bar{\gamma}+\tilde{\gamma})$ is a hedging strategy for the contingent claim $g\left(S_{T}\right)$ with lower bound $\underline{\Gamma}$ on the gamma process. Conversely, given a hedging strategy of $g\left(S_{T}\right)$ which satisfies the lower bound $\underline{\Gamma}$ on the gamma process, it is possible to decompose it as above. In view of this, we shall assume in the rest of this section that $\underline{\Gamma}=0$ without loss of generality.

\subsection{Hedging under a non-negativity constraint on the gamma}

We now concentrate on the reduced problem of hedging some contingent claim $g(S(T))$ with bounds on the gamma process given by

$$
\bar{\Gamma}=+\infty \text { and } \underline{\Gamma}=0 .
$$

In this case, the dynamic programming equation is

$$
-\hat{V}_{t}-\frac{1}{2} \sigma^{2} s^{2}\left(\hat{V}_{s s}\right)^{+}=0 .
$$

Formally the hedging strategy is simple. Let

$$
\mathcal{C}:=\{(t, s) \in[0, T) \times(0, \infty): \hat{V} \text { is locally convex at }(t, s)\},
$$

so that on $\mathcal{C}, V$ solves the Black-Scholes PDE and therefore, is smooth. (A precise definition of $\mathcal{C}$ is given in (7.3) below.) On this region, we follow the Black-Scholes hedge, i.e., on $\mathcal{C}$ we take

$$
Y(r)=\hat{V}_{s}(r, S(r))
$$

This is achieved by taking

$$
\alpha(r)=\mathcal{L} \hat{V}_{s}(r, S(r)), \quad \text { and } \quad \gamma(r)=\hat{V}_{s s}(r, S(r)) .
$$

Outside $\mathcal{C}$, we follow a buy and hold strategy. Indeed, if $(t, s) \notin \mathcal{C}$, let

$$
y_{0}=\hat{V}_{s}(t, s)
$$


and choose $\alpha \equiv \gamma \equiv 0$, i.e., we take $Y(r) \equiv y_{0}$. We follow, this strategy until the first stopping time

$$
\theta(t):=T \wedge \inf \{r>t: \hat{V}(r, S(r))>X(r)\} .
$$

Note that for $r \in[t, \theta], Y \equiv y_{0}=\hat{V}_{s}(t, s)$ and

$$
X(r)=\hat{V}(t, s)+y_{0}[S(r)-s]=\hat{V}(t, s)+\hat{V}_{s}(t, s)[S(r)-s] .
$$

Recalling from Remark 6.1 that $\hat{V}=g$ outside $\mathcal{C}$, we see that

$$
\begin{aligned}
X(r) & =g(s)+\hat{g}_{s}(s)[S(r)-s] \\
& \geq g(S(r)) \\
& =\hat{V}(r, S(r)) \quad \text { whenever }(u, S(u)) \notin \mathcal{C} \text { for all } u \in[t, r),
\end{aligned}
$$

where the inequality follows from the concavity of $\hat{V}=g$ outside $\mathcal{C}$. Hence, we expect $\hat{V}$ to satisfy $\hat{V}(r, S(r)) \leq X(r)$ as long as $(r, S(r))$ stay outside of $\mathcal{C}$ and possibly longer.

Clearly, when the above hedging strategy exists it satisfies the lower gamma bound. On the region of convexity $\mathcal{C}, \hat{V}_{s s}$ is non-negative and outside the $\mathcal{C}$ the buy and hold strategy has zero gamma. Jump part of the portfolio $Y$ is needed when we switch between the buy and hold strategy and the Black-Scholes hedge.

At the end of the section, we prove a verification type result, Theorem 7.1. However, before the theorem we give the following simple example to illustrate the construction.

Example 7.1 Consider the payoff $g$ with the properties that

$$
g(s)=1, \text { for } s \geq 1, g(0)=0, g \text { is non-decreasing and convex on }[0,1] .
$$

We claim that the minimal super-replicating price $\hat{V}$ with the above claim and $\underline{\Gamma}>0$, $\bar{\Gamma}=\infty$, satisfies

$$
\hat{V}(t, s)=1, \text { for } s \geq 1, \hat{V}(t, 0)=0, \quad \hat{V}(t, \cdot) \text { is non-decrasing and convex on }[0,1],
$$

and on $[0,1], \hat{V}$ is the unique solution of

$$
-\hat{V}_{t}-\frac{1}{2} \sigma^{2} s^{2} \hat{V}_{s s}=0, \quad s \in(0,1),
$$

together with the boundary conditions

$$
\hat{V}(t, 0)=0, \quad \hat{V}(t, 1)=1, \quad \hat{V}(T, s)=g(s) .
$$

Indeed, we directly check that $\hat{V}$ defined as above is a viscosity solution of the dynamic programming equation (7.1) on the whole domain. Therefore, it is the minimal superreplication price. Notice that at the point $s=1$, one needs to use the theory of viscosity solutions as the function $V$ is not differentiable at that point. 
The hedging strategy is constructed as follows. For $s \geq 1$, we place one dollar in the bond. Then, $X(T)=1 \geq g(S(T))$. For $s \leq 1$, we use the Black-Scholes hedge until $S(t)=1$. Afterwards, we set $Y \equiv 0$. Precisely, let $\theta \geq 0$ be the time at which the stock price is greater than one, i.e. $S_{s, t}(\theta) \geq 1$. Then,

$$
Y(r)=\left\{\begin{array}{cc}
\hat{V}_{s}\left(r, S_{s, t}(r)\right), & r \in[0, \theta] \\
0, & r \geq \theta
\end{array}\right.
$$

The above portfolio process satisfies the non-negative gamma constraint. Indeed, on $[0, \theta]$, $\gamma(r)=\hat{V}_{s s}\left(r, S_{s, t}(r)\right) \geq 0$ and on $r \geq \theta, \gamma(r)=0$. Notice that, at $r=\theta, Y$ may experience a jump, which is allowed in the model. However, the process $\gamma$ is not of the form (2.4) so that it is not admissible in the strict sense of Section 2.

Finally, we remark on the formal connection between $V_{s s}$ and $\gamma$. Formally, if one expects that these two quantities are equal, then the constraint $\gamma \geq-\underline{\Gamma}$ translates to a differential inequality $V_{s s} \geq-\underline{\Gamma}$. However, in this example $V$ does not satisfy this condition globally. So it is clear that the formal argument that equates $\gamma$ to $V_{s s}$ does not always hold.

Our next result is an analogue of the classical verification theorem in the optimal control theory. Set

$$
\mathcal{C}[\hat{V}]:=\left\{(t, s): \liminf _{h \rightarrow 0,\left(t^{\prime}, s^{\prime}\right) \rightarrow(t, s)} \frac{\hat{V}\left(t^{\prime}, s^{\prime}+h\right)+\hat{V}\left(t^{\prime}, s^{\prime}-h\right)-2 \hat{V}\left(t^{\prime}, s^{\prime}\right)}{h^{2}}>0\right\} .
$$

Then, $\mathcal{C}[\hat{V}]$ is an open set and it is the precise definition of the set $\mathcal{C}$ introduced earlier in (7.2). By, (7.1), we see that

$$
-\hat{V}_{t}-\frac{1}{2} \sigma^{2} s^{2} \hat{V}_{s s}=0, \text { on } \mathcal{C}[\hat{V}]
$$

Theorem 7.1 (Verification) Suppose that there exists an admissible control $\hat{\nu}=\left(\left(\hat{\tau}_{n}, \hat{y}_{n}\right)_{n>0}, \hat{\alpha}, \hat{\gamma}\right)$ satisfying $\tau_{0}=t$,

$$
\begin{gathered}
\tau_{2 n+1}=T \wedge \inf \left\{r \geq \tau_{2 n}:\left(r, S_{t, s}(r)\right) \notin \mathcal{C}[\hat{V}]\right\}, \quad n \geq 0, \\
\tau_{2 n}=T \wedge \inf \left\{r \geq \tau_{2 n-1}: \hat{V}\left(r, S_{t, s}(r)\right)-\hat{V}\left(\tau_{2 n-1}, S_{t, s}\left(\tau_{2 n-1}\right)\right)\right. \\
\left.\quad>Y\left(\tau_{2 n-1}\right)\left[S_{t, s}(r)-S_{t, s}\left(\tau_{2 n-1}\right)\right]\right\}, \quad n \geq 1, \\
Y(r)=\hat{V}_{s}\left(r, S_{t, s}(r)\right), r \in\left[\tau_{2 n}, \tau_{2 n+1}\right), \quad n \geq 0, \\
Y(r)=Y\left(\tau_{2 n-1}, S_{t, s}\left(\tau_{2 n-1}\right)\right), r \in\left[\tau_{2 n-1}, \tau_{2 n}\right), \quad n \geq 1 .
\end{gathered}
$$

Then, $\hat{\nu}$ is super-replicating.

Proof. Let $\hat{X}$ be the wealth process corresponding to the investment strategy $\hat{\nu}$ with initial wealth $\hat{X}(t)=\hat{V}(t, s)$. Suppose that we have proved that

$$
\hat{X}\left(\tau_{2 n}\right)=\hat{V}\left(\tau_{2 n}, S_{t, s}\left(\tau_{2 n}\right)\right)
$$


for some $n \geq 0$. Then, on $\left[\tau_{2 n}, \tau_{2 n+1}\right)$ the process $\left(r, S_{t, s}(r)\right) \in \mathcal{C}[\hat{V}]$ and $Y(r)=$ $\hat{V}_{s}\left(r, S_{t, s}(r)\right)$. Since $\hat{V}$ satisfies (7.4), a standard application of Itô's rule yields

$$
\hat{X}\left(\tau_{2 n+1}\right)=\hat{V}\left(\tau_{2 n+1}, S_{t, s}\left(\tau_{2 n+1}\right)\right) .
$$

For $r \in\left[\tau_{2 n+1}, \tau_{2 n+2}\right)$,

$$
Y(r)=Y\left(\tau_{2 n+1}\right)
$$

Hence,

$$
\hat{X}(r)=\hat{V}\left(\tau_{2 n+1}, S_{t, s}\left(\tau_{2 n+1}\right)\right)+Y\left(\tau_{2 n+1}\right)\left[S_{t, s}(r)-S_{t, s}\left(\tau_{2 n+1}\right)\right] .
$$

By the definition of $\tau_{2 n+2}$, we immediately conclude that

$$
\hat{X}\left(\tau_{2 n+2}\right)=\hat{V}\left(\tau_{2 n+2}, S_{t, s}\left(\tau_{2 n+2}\right)\right) \quad \text { on } \quad\left\{\tau_{2 n+2}<T\right\},
$$

and

$$
\hat{X}\left(\tau_{2 n+2}\right) \geq \hat{V}\left(\tau_{2 n+2}, S_{t, s}\left(\tau_{2 n+2}\right)\right) \quad \text { on } \quad\left\{\tau_{2 n+2}=T\right\}
$$

Now by induction, we conclude that $\hat{\nu}$ is super-replicating.

We conclude this section by some remarks.

Remark 7.1 The conditions in the statement of the above theorem, give the construction of $Y$. We only need to describe the choice of $Y\left(\tau_{2 n+1}\right)$ for all $n=1,2, \ldots$. Once this choice is made, on $\left[\tau_{2 n}, \tau_{2 n+1}\right)$ the process $\left(r, S_{t, s}(r)\right) \in \mathcal{C}[\hat{V}]$ and $Y(r)=\hat{V}_{s}\left(r, S_{t, s}(r)\right)$. This means that we have to choose

$$
\begin{aligned}
& \alpha(r)=\mathcal{L} \hat{V}_{s}\left(r, S_{t, s}(r)\right)=\hat{V}_{t, s}\left(r, S_{t, s}(r)\right)+\frac{1}{2} \sigma^{2} S_{t, s}(r)^{2} \hat{V}_{s s}\left(r, S_{t, s}(r)\right) \\
& \gamma(r)=\hat{V}_{s s}\left(r, S_{t, s}(r)\right) .
\end{aligned}
$$

By standard parabolic regularity, $\hat{V}$ is smooth on $\mathcal{C}[\hat{V}]$. Hence, at least locally, $\alpha$ and $\gamma$ are well defined.

Moreover, on $\left[\tau_{2 n+1}, \tau_{2 n+2}\right)$ we take $\alpha \equiv \gamma \equiv 0$.

Provided that the stopping times do not accumulate, this construction will always yield a strategy which satisfies the gamma constraint (2.5). Other obstacles in ensuring the admissibility of this control are of technical nature.

The choice of $Y\left(\tau_{2 n+1}\right)$ is crucial in order to make sure that the resulting strategy is defined and is also admissible. The chief difficulty is to ensure that the stopping times do not accumulate. Recall that the stopping time $\tau_{2 n+1}$ is the exit time from $\mathcal{C}[\hat{V}]$. In all the examples, we see that at the boundary of the region of convexity $\mathcal{C}[\hat{V}], \hat{V}_{s}$ has a concave jump discontinuity. Or equivalently, at any $(t, s)$ at the boundary of $\mathcal{C}[\hat{V}]$, there is $p \in \mathbb{R}$ in the subdifferential of $V$ so that

$$
\hat{V}\left(t, s^{\prime}\right) \leq \hat{V}(t, s)+p\left(s^{\prime}-s\right)
$$


for all $s^{\prime}$ close to $s$. If there are $p$ 's satisfying the above inequality, we choose $Y\left(\tau_{2 n+1}\right)$ to be an interior point of all such $p$ 's.

Remark 7.2 In general, the investment strategy outlined in this section may not be feasible to implement as the stopping times defined may accumulate. It may not even be possible to prove the optimality or the existence of the formal optimal strategy in the general set-up. This difficulty is in analogy with the construction of optimal feedback strategies in standard optimal control. There the formal optimal feedback control is given as the maximizing control in the dynamic programming equation. It is well known that, although in general it is not possible to prove the optimality or the existence of this feedback policy, in many applications it has been used successfully.

\section{A normalized gamma constraint}

In this section, we briefly look at the problem obtained by replacing the constraint (2.5) by

$$
-\underline{\Gamma} X(r) \leq S_{t, s}(r)^{2} \gamma(r) \leq \bar{\Gamma} X(r) \text { for all } r \in[t, T]
$$

where $X$ is the wealth process defined in (2.2). As discussed earlier, since $\gamma$ formally corresponds to $V_{s s}$ and $X$ to $V$, the above constraint is an attempt to control the ratio $s^{2} V_{s s} / V$. Moreover, a dimension analysis indicate that the ratio is dimensionless and therefore, it is a more natural quantity to control.

An analysis almost identical to the one given in the constraint (2.5) yields the following representation results for the value function with this new constraint (8.1).

Theorem 8.1 Let $V$ be the value function with the constraint (8.1). Suppose that $g$ satisfies (2.6), and define

$$
\hat{g}(s):=\sup _{\tau \in \mathcal{T}_{t}^{\infty}} \mathbb{E}\left[e^{-\frac{1}{2} \sigma^{2} \bar{\Gamma} \tau} g\left(S_{t, s}(\tau)\right)\right] .
$$

Then, for all $t<T$,

$$
\begin{aligned}
V(t, s) & =\sup _{\theta \in \mathcal{T}_{t}^{T}, \tau \in \mathcal{T}_{\theta}^{\infty}} \mathbb{E}\left[e^{-\frac{1}{2} \sigma^{2}(\bar{\Gamma}(\tau-\theta)+\underline{\Gamma}(T-\theta))} g\left(S_{t, s}(\tau)\right)\right] \\
& =\sup _{\theta \in \mathcal{T}_{t}^{T}} \mathbb{E}\left[e^{-\frac{1}{2} \sigma^{2}(\underline{\Gamma}(T-\theta))} \hat{g}\left(S_{t, s}(\tau)\right)\right] \\
& =\sup _{x \in \mathcal{X}} \mathbb{E}\left[e^{-\frac{1}{2} \underline{\Gamma} \sigma^{2} \int_{t}^{T} x(r) d r} \hat{g}\left(S_{t, s}^{x}(T)\right)\right] \\
& =\sup _{(x, y) \in \mathcal{X} \times \mathcal{Y}} \mathbb{E}\left[e^{-\frac{1}{2} \sigma^{2} \int_{t}^{T}(\underline{\Gamma} x(r)+\bar{\Gamma} y(r)) d r} \hat{g}\left(\hat{S}_{t, s}^{x, y}(T)\right)\right] .
\end{aligned}
$$




\section{References}

[1] Black F. and M. Scholes (1973). The pricing of options and corporate liabilities. Journal of Political Economy 81, 637-659.

[2] Broadie M., Cvitanić J. and Soner H.M. (1998). Optimal replication of contingent claims under portfolio constraints. The Review of Financial Studies 11, 59-79.

[3] Cheridito P., Soner H.M. and Touzi N. (2003a). The multi-dimensional superreplication problem under gamma constraints. Annales de L'Instititut Henri Porincare Analyse Non Lineaire, forthcoming.

[4] Cheridito P., Soner H.M. and Touzi N. (2003b). Small time path behavior of double stochastic integrals and applications to stochastic control. Preprint.

[5] Cvitanić J. and Karatzas I. (1993). Hedging contingent claims with constrained portfolios. Annals of Applied Probability 3(3), 652-681.

[6] Fleming W.H. and Soner H.M. (1993). Controlled Markov Processes and Viscosity Solutions. Applications of Mathematics, 25. Springer-Verlag, New York.

[7] Karatzas I. and Shreve S. (1991). Brownian Motion and Stochastic Calculus, Second Edition. Graduate Texts in Mathematics, 113. Springer-Verlag, New York.

[8] Karatzas I. and Shreve S.E. (1998). Methods of Mathematical Finance, SpringerVerlag, New York, Heidelberg, Berlin.

[9] Lions, P.-L. (1983). Optimal Control of Diffusion Processes and Hamilton-JacobiBellman Equations, Parts I and II Communications in P.D.E. 8, 1101-1174, 12291276 .

[10] Soner H.M. (2005) Stochastic Optimal Control in Finance, Cattedra Galileiana April 2003, Scuola Normale, Pisa.

[11] Soner H.M., Shreve S.E. and Cvitanić J. (1995). There is no nontrivial hedging portfolio for option pricing with transaction costs, Annals of Applied Prob., 5/2, 327-355.

[12] Soner H.M., and Touzi N. (2000). Super-replication under gamma constraints. SIAM J.Control and Opt. 39(1), 73-96. 